\title{
Violência interpessoal contra a mulher no Estado do Ceará de 2009 a 2018: estudo
}

\section{ecológico}

\author{
Interpersonal violence against women in the State of Ceará from 2009 to 2018: an ecological study \\ Violencia interpersonal contra la mujer en el Estado de Ceará de 2009 a 2018: un estudio ecológico
}

Recebido: 13/08/2021 | Revisado: 19/08/2021 | Aceito: 23/08/2021 | Publicado: 24/08/2021

Paola Rachel Pinheiro Leitão

ORCID: https://orcid.org/0000-0002-3515-7740

Universidade de Fortaleza, Brasil

E-mail: paolarachelleitao@gmail.com

Jefferson Felipe Calazans Batista

ORCID: https://orcid.org/0000-0002-3681-7990

Universidade Tiradentes, Brasil

E-mail: jefferson.calazans.enf@gmail.com

Brenda Layssa Lima Dantas

ORCID: https://orcid.org/0000-0002-4403-1496

Universidade Tiradentes, Brasil

E-mail: brenda.layssa@hotmail.com

Ana Vitória do Nascimento Oliveira

ORCID: https://orcid.org/0000-0003-1008-5688

Universidade de Fortaleza, Brasil

E-mail: vitoriaannahnascimento@gmail.com

Silvia Rebeca Sabóia Quezado

ORCID: https://orcid.org/0000-0001-6252-2531

Universidade Federal do Ceará, Brasil

E-mail: rebecaquezado@hotmail.com

Juliana de Oliveira Musse Silva

ORCID: https://orcid.org/0000-0003-1713-6791

Universidade Federal de Sergipe, Brasil E-mail: julimusse@hotmail.com

\begin{abstract}
Resumo
Objetivo: Analisar a tendência da violência interpessoal contra a mulher no Estado do Ceará de 2009 a 2018. Métodos: Trata-se de um estudo ecológico de série temporal, sobre os casos notificados e mortalidade por violência contra mulher. Foram selecionadas quatro grupos de variáveis para análise: perfil da vítima/caso, tipos de violência, mecanismos de ação e agressor. Para análise de tendência adotou-se o modelo de regressão linear simples. Resultados: De 2009 a 2018, foram 19.135 casos de violência contra a mulher. Ao longo dos anos observou-se um aumento de $95,1 \%$ no quantitativo de notificações. Verificou-se associação estatisticamente significativa entre as taxas de mortalidade e os anos do estudo $(\mathrm{p}=0,0024)$, indigitando um incremento anual de 0,5885. O coeficiente de prevalência mostrou um aumento anual relevante - de 11,5188 ( $\mathrm{p}<0,001$ ). Conclusão: No decorrer da série temporal os coeficientes de prevalência aumentaram, o que demonstra a efetividade de algumas políticas de saúde públicas direcionadas para esse aspecto.
\end{abstract}

Palavras-chave: Mulheres; Homicídio; Violência contra mulher; Notificação.

\begin{abstract}
Objective: To analyze the trend of interpersonal violence against women in the State of Ceará from 2009 to 2018. Methods: This is an ecological time series study on reported cases and mortality from violence against women. Four groups of variables were selected for analysis: profile of the victim/case, types of violence, mechanisms of action and aggressor. For trend analysis, the simple linear regression model was adopted. Results: From 2009 to 2018, there were 19,135 cases of violence against women. Over the years, there was an increase of $95.1 \%$ in the number of notifications. There was a statistically significant association between mortality rates and years of study $(\mathrm{p}=0.0024)$, indicating an annual increase of 0.5885 . The prevalence coefficient showed an annual increase - relevant - of 11.5188 ( $\mathrm{p}<0.001)$. Conclusion: During the time series, the prevalence coefficients increased, which demonstrates the effectiveness of some public health policies aimed at this aspect.
\end{abstract}

Keywords: Women; Homicide; Violence against women; Notification.

\section{Resumen}

Objetivo: Analizar la tendencia de la violencia interpersonal contra las mujeres en el estado de Ceará de 2009 a 2018. Métodos: Se trata de un estudio de serie temporal ecológica sobre casos reportados y mortalidad por violencia contra 
las mujeres. Se seleccionaron cuatro grupos de variables para el análisis: perfil de la víctima/caso, tipos de violencia, mecanismos de acción y agresor. Para el análisis de tendencias, se adoptó el modelo de regresión lineal simple. Resultados: De 2009 a 2018 se registraron 19.135 casos de violencia contra la mujer. A lo largo de los años, hubo un aumento del 95,1\% en el número de notificaciones. Hubo una asociación estadísticamente significativa entre las tasas de mortalidad y los años de estudio $(\mathrm{p}=0,0024)$, lo que indica un aumento anual de 0,5885 . El coeficiente de prevalencia mostró un incremento anual - relevante - de 11,5188 ( $\mathrm{p}<0,001$ ). Conclusión: Durante la serie de tiempo, los coeficientes de prevalencia aumentaron, lo que demuestra la efectividad de algunas políticas de salud pública orientadas a este aspecto.

Palabras clave: Mujeres; Asesinato; Violencia contra las mujeres; Notificación.

\section{Introdução}

A violência contra a mulher é qualquer conduta, ação ou omissão discriminatória, ataque ou coerção, que cause danos, morte, constrangimento, limitação, sofrimento físico, sexual, moral, psicológico, social, político, econômico ou perda patrimonial (Souza, 2009). É considerada como transgressão ao direito natural imposto pelo ordenamento jurídico pátrio, que protege os direitos fundamentais da pessoa humana e equipara homens e mulheres em direitos e obrigações.

O Brasil demonstrou 1.151 e 1.206 casos de feminicídio, em 2017 e 2018, respectivamente (crescimento de 4\%). A maior parte das vítimas é de mulheres negras (61\%) e em idade reprodutiva (28,2\% das vítimas tinham de 20 a 29 anos, 29,8 tinham de 30 e 39 anos e 18,5\% tinham de 40 a 49 anos). Quanto aos agressores, destaca-se o fato de que 88,8\% eram companheiros ou ex-companheiros das vítimas. Vale ressaltar que, em 2018, mais de 263 mil casos de lesão corporal dolosa ocorreram contra o público feminino, sendo 5.170 destes somente no Ceará. Este, ainda no mesmo ano, foi o segundo Estado com o maior índice de feminicídio (448 casos) (Brasil \& Fórum Brasileiro de Segurança Pública, 2019).

Em razão da complexidade dessa conjunção de problemas, iniciativas governamentais direcionadas à proteção da mulher, por meio da formulação e execução de políticas públicas, são desenvolvidas no decorrer dos anos. Com efeito, exprimese que a violência é um aspecto que transcende a esfera da segurança pública, permeando outros setores, inclusive o da saúde. Os serviços de saúde, frequentemente, acolhem pessoas acometidas por diversas situações de violência. A equipe multidisciplinar nesses ambientes tem potencial para realizar um atendimento humanizado e integral, privilegiando a complexidade da situação e as múltiplas consequências impostas às vítimas, motivo pelo qual a integralidade e a interdisciplinaridade são fundamentais (Brasil \& Ministério da Saúde, 2012; Paraná \& Secretaria de Estado da Saúde do Paraná, 2017).

A pesquisa teve como objetivo analisar a tendência da violência interpessoal contra a mulher no Estado do Ceará de 2009 a 2018.

\section{Metodologia}

Este ensaio conforma um estudo ecológico de série temporal, de abordagem quantitativa e caráter descritivo, analítico e exploratório, que avaliou os dados sobre violência interpessoal e mortalidade por agressões contra a mulher no Estado do Ceará de 2009 a 2018. Este tipo de estudo segundo Lima-Costa e Barreto (2003) tem a capacidade de observar a ocorrência de um agravo ou condição relacionada à saúde e comparar com a exposição de interesse entre agregados de indivíduos, podendo assim verificar uma possível associação entre eles.

As informações foram colhidas do Sistema de Informações de Agravos e Notificações (SINAN), referentes à seção sobre violência interpessoal, e do Sistema de Informação sobre Mortalidade (SIM), disponíveis no Departamento de Informática do Sistema Único de Saúde (DATASUS) (Brasil \& Departamento de Informática do Sistema Único de Saúde, 2020).

As variáveis categóricas referentes ao perfil da violência utilizadas para análise estão descritas no Quadro 1. 
Quadro 1 - Variáveis categóricas referentes ao perfil da violência selecionados para análise, Fortaleza, Ceará.

\begin{tabular}{|c|c|c|c|}
\hline \multicolumn{4}{|c|}{ Variáveis } \\
\hline Perfil do caso/vítima & Tipos de violência & Mecanismo de ação & Agressor \\
\hline Faixa etária & Violência física & Força corporal/espancamento & Pai \\
\hline Raça & Violência psico/moral & Enforcamento & Mãe \\
\hline Escolaridade & Tortura & Objeto contundente & Padrasto \\
\hline Local da ocorrência & Violência sexual & Objeto perfuro-cortante & Madrasta \\
\hline Evolução do caso & & Envenenamento & Cônjuge/ Ex-cônjuge \\
\hline Religião & & Arma de fogo & Namorado/Ex-namorado \\
\hline & & & Filha \\
\hline & & & Irmão \\
\hline & & & Amigos \\
\hline & & & Cuidador \\
\hline & & & Desconhecido \\
\hline & & & Chefe/patrão \\
\hline & & & Policial \\
\hline
\end{tabular}

Fonte: DATASUS.

Para o cálculo de taxa de incidência (TI) e taxa de mortalidade (TM), recorreu-se ao Censo Populacional de 2010, como também foram utilizadas as projeções censitárias de 2009 a 2018, provenientes do Instituto Brasileiro de Geografia e Estatística (IBGE).

A TI foi calculada por meio da fórmula:

$$
T I=\frac{N^{\circ} \text { de casos de violência contra mulher em um local e período }}{\text { População feminina no mesmo local e período }} \times 100.000 .
$$

Para coleta dos dados sobre mortalidade por violência em mulheres, o código X85 a Y09 da Classificação Internacional de Doenças (CID-10) foi utilizado, agregando-se as variáveis sexo feminino e os anos de 2009 a 2018. Com efeito, o cálculo da TM deu-se por:

$$
T M=\frac{N^{\circ} \text { de mortes por agressão em mulheres no mesmo local e período }}{\text { População feminina no mesmo local e período }} \times 100.000 \text {. }
$$

Este estudo apropriou-se da estatística descritiva e analítica. Os dados foram dispostos por meio de frequência absoluta e relativa, média, amplitude, mínimo e máximo. Para as análises de tendências (por ano), foram utilizadas as taxas de notificações/taxas de mortalidade, sendo estas a variável dependente (y), e os respectivos anos como variável independente (x).

Optou-se pelo cálculo de regressão linear simples $Y^{\prime}=a+b X$, sendo este somente aplicado em caso de comprovação da normalidade dos dados, mediante aplicação do teste de Shapiro-Wilk. Foi considerado estatisticamente significativo p $<0,05$. 
Research, Society and Development, v. 10, n. 11, e97101119400, 2021

(CC BY 4.0) | ISSN 2525-3409 | DOI: http://dx.doi.org/10.33448/rsd-v10i11.19400

Para a disposição, organização e análise descritiva dos dados, recorreu-se ao Microsoft Excel 2020, enquanto, para efetivar os cálculos de regressão linear simples, apelou-se para o programa BioStat 5.3.

\section{Resultados}

O Estado do Ceará regista uma densidade populacional alta, sendo constituída por mais de quatro milhões de mulheres. De 2009 a 2018 foi constatado o total de 19.135 casos de violência contra a mulher, notificados no SINAN. O primeiro ano analisado (2009) apontou 1,93\% (n=292) de casos, que, em comparação ao último, com 31,2\% ( $\mathrm{n}=5.969)$, demonstrou aumento de 95,1\% no quantitativo de notificações. A maioria das mulheres vítimas de violência interpessoal do estudo era de pardas (74,1\%), com faixa etária de dez a 19 anos (28,8\%) e que cursaram a $5^{\mathrm{a}}$ e $8^{\mathrm{a}}$ séries incompletas do ensino fundamental (17,4\%) e eram acometidas, principalmente, no ambiente domiciliar (65,8\%) (Tabela 1). 
Research, Society and Development, v. 10, n. 11, e97101119400, 2021

(CC BY 4.0) | ISSN 2525-3409 | DOI: http://dx.doi.org/10.33448/rsd-v10i11.19400

Tabela 1 - Perfil da vítima/caso de violência contra a mulher no Ceará, de 2009 a 2018.

\begin{tabular}{|c|c|c|}
\hline Perfil da vítima/caso & $\mathbf{N}$ & $\%$ \\
\hline \multicolumn{3}{|l|}{ Raça/cor } \\
\hline Branca & 2716 & 14,2 \\
\hline Preta & 1025 & 5,4 \\
\hline Amarela & 123 & 0,6 \\
\hline Parda & 14189 & 74,1 \\
\hline Indígena & 82 & 0,4 \\
\hline \multicolumn{3}{|l|}{ Escolaridade } \\
\hline Analfabeta & 537 & 2,8 \\
\hline $1^{\mathrm{a}} \mathrm{a} 4^{\mathrm{a}}$ série incompleta do EF & 1587 & 8,3 \\
\hline $4^{\mathrm{a}}$ série completa do EF & 745 & 3,9 \\
\hline $5^{\mathrm{a}}$ a $8^{\mathrm{a}}$ série incompleta do $\mathrm{EF}$ & 3340 & 17,4 \\
\hline Ensino fundamental completo & 1099 & 5,7 \\
\hline Ensino médio incompleto & 1705 & 8,9 \\
\hline Ensino médio completo & 2040 & 10,7 \\
\hline Educação superior incompleta & 544 & 2,8 \\
\hline Educação superior completa & 425 & 2,2 \\
\hline \multicolumn{3}{|l|}{ Local da ocorrência } \\
\hline Residência & 12592 & 65,8 \\
\hline Habitação Coletiva & 107 & 0,6 \\
\hline Escola & 250 & 1,3 \\
\hline Local de pratica esportiva & 44 & 0,2 \\
\hline Bar ou Similar & 291 & 1,5 \\
\hline Via pública & 2863 & 15,0 \\
\hline Comércio/Serviços & 162 & 0,8 \\
\hline Indústrias/construção & 22 & 0,1 \\
\hline Outros & 1478 & 7,7 \\
\hline \multicolumn{3}{|l|}{ Faixa etária } \\
\hline 0 a 9 anos & 2560 & 13,4 \\
\hline 10 a 19 anos & 5519 & 28,8 \\
\hline 20 a 29 anos & 4031 & 21,1 \\
\hline 30 a 39 anos & 3102 & 16,2 \\
\hline 40 a 49 anos & 1841 & 9,6 \\
\hline 50 a 59 anos & 790 & 4,1 \\
\hline 60 e mais & 1279 & 6,7 \\
\hline
\end{tabular}

Fonte: DATASUS.

Quanto ao tipo de agressão, as mulheres sofriam, principalmente, a violência física (54,8\%), seguida da violência psicológica/moral (35,5\%). Entrementes, os mecanismos de ação mais utilizados foram a força corporal/espancamento (35,3\%), seguidos do emprego de objeto perfurocortante $(9,1 \%)$. No que concerne aos agressores, esses eram cônjuges ou ex-cônjuges $(25 \%)$, mães $(14,1 \%)$ e pais $(10,4 \%)$ (Tabela 2$)$. 
Tabela 2 - Tipo de violência, mecanismo de ação e agressor dos casos de violência contra mulher no Ceará, de 2009 a 2018.

\begin{tabular}{lcc}
\hline Tipo de violência & $\mathbf{N}$ & \% \\
\hline Violência física & 10485 & 54,8 \\
Violência psico/moral & 6807 & 35,5 \\
Tortura & 768 & 4,0 \\
Violência sexual & 3403 & 17,8 \\
\hline Mecanismo de ação & & 35,3 \\
\hline Força corporal/espancamento & 6760 & 4,5 \\
Enforcamento & 862 & 4,3 \\
Objeto contundente & 821 & 9,1 \\
Objeto perfurocortante & 1736 & 4,8 \\
Arma de fogo & 923 & 10,4 \\
\hline Agressor (a) & & 14,1 \\
Pai & 1984 & 1,4 \\
Mãe & 2704 & 0,1 \\
Padrasto & 262 & 25,0 \\
Madrasta & 25 & 7,6 \\
Cônjuge/ex & 4779 & 4,4 \\
Namorado/ex & 1456 & 2,8 \\
Filho (a) & 846 & 9,6 \\
Irmão & 542 & 0,7 \\
Amigos & 1841 & 9,8 \\
Cuidador & 140 & 0,1 \\
Desconhecido & 1868 & 0,5 \\
Chefe/patrão & 26 & \\
Policial & 99 & \\
\hline
\end{tabular}

Fonte: DATASUS.

Os dados referentes às taxas de incidência e taxa de mortalidade demonstraram aumento no decorrer dos anos, com destaque para a primeira, que teve elevação de 94,8\% nos casos de violência contra a mulher. O ano de 2018 exibiu o maior valor dentre ambos coeficientes, sendo 127,8 casos para cada 100 mil mulheres (TI) e 10,2 óbitos por violência para cada 100 mil mulheres (TM). Vale ressaltar que, referente à TM, os anos de 2015 e 2016 expressaram notória redução, de 5,6/100 mil e 4,7/100 mil mulheres, reascendendo em 2017, com 8,1 mortes/100 mil (Gráfico 1). 
Gráfico 1 - Taxa de incidência e taxa de mortalidade por violência contra a mulher no Ceará, de 2009 a 2018.

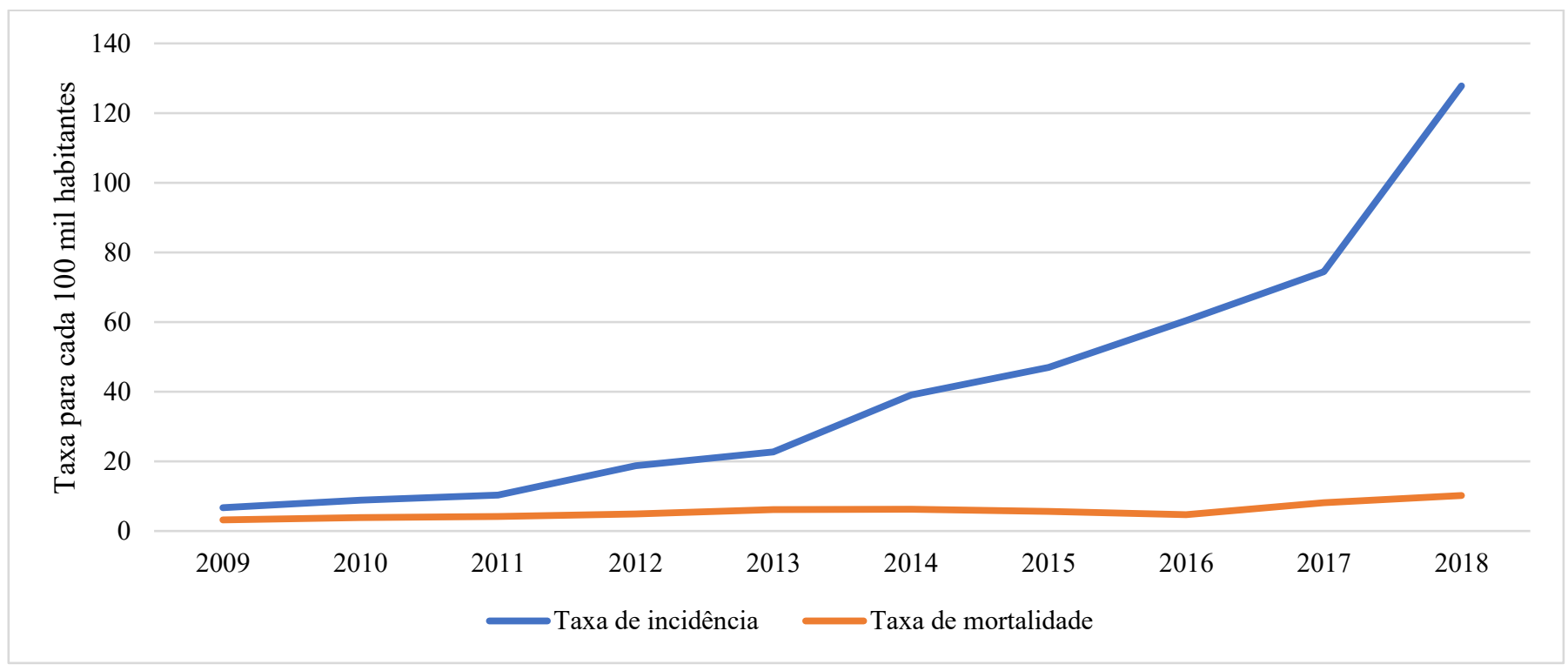

Fonte: DATASUS.

No tocante à análise de tendência, verificou-se associação estatisticamente significativa entre as taxas de mortalidade e os anos do estudo $(\mathrm{p}=0,0024)$, indigitando um incremento anual de 0,5885 . A taxa de incidência mostrou um aumento anual relevante - de 11,5188 (p=0,0004) (Tabela 3).

Tabela 3 - Tendência da mortalidade e dos casos de violência contra a mulher no Ceará, de 2009 a 2019.

\begin{tabular}{lccccc}
\hline \multirow{2}{*}{ Taxa } & \multicolumn{3}{c}{ Regressão linear simples } \\
\cline { 2 - 6 } & \multicolumn{2}{c}{$\mathrm{R}^{2}$} & $\mathrm{a}$ & $\mathrm{b}$ & $p$ valor $^{\mathrm{a}}$ \\
\hline Mortalidade & 0,6785 & $-1179,1842$ & 0,5885 & 0,0024 \\
Casos & 0,8190 & $-23151,45$ & 11,5188 & 0,0004 \\
\hline
\end{tabular}

Nota: $\mathrm{R}^{2}=$ Coeficiente de determinação (ajustado); $\mathrm{a}=$ Intercepto (estimação do modelo para mortalidade e dos casos ao ano zero de observação); $\mathrm{b}=$ Coeficiente de regressão (incremento da de mortalidade e casos para cada acréscimo de 1 ano); $p$ valor $=$ Teste $\mathrm{F}($ ANOVA). Fonte: Dados da pesquisa (2021).

\section{Discussão}

Os resultados da pesquisa apontam um perfil de mulheres que sofreram violência como sendo jovens, pardas, com baixa escolaridade e que são vítimas dos companheiros e em ambiente doméstico. No período de 2017 a 2018, os dados do Instituto de Pesquisa Econômica Aplicada (IPEA) (Instituto De Pesquisa Econômica Aplicada, 2020) apontaram que o Ceará estava entre as Unidades da Federação (UFs) cujas taxas de homicídios de mulheres mais aumentaram no período (aumento superior a 20\%). Apenas no ano de 2017, das 374 mulheres mortas no Ceará, 202 estavam na faixa etária de 15 a 29 anos, possuindo um dos maiores indicadores no quesito morte entre jovens mulheres, perdendo apenas para o Estado da Bahia (208 casos). No que concerne a raça/cor, entretanto, os dados do mapa apontaram que mulheres negras morrem mais quando comparadas às não negras, ficando o Estado com o segundo lugar (325 casos), quando comparado às demais unidades federativas nacionais.

O Estado, em 2018, registrou uma das maiores taxas de homicídio feminino por 100 mil habitantes (20,5 e 10,2); e as taxas de homicídios de mulheres negras foram quase quatro vezes maiores do que os percentuais das não negras.

Demanda efetivada pela Defensoria Pública do Estado do Ceará, no período de 2016 a 2019, com mulheres vítimas de violência, que buscaram assistência no Núcleo de Enfrentamento à Violência Contra a Mulher (NUDEM), em Fortaleza, 
demonstrou o perfil dos agressores como ex-companheiros e os cônjuges, com $47 \%$ e 36\%, respectivamente, e que, em $42 \%$ dos casos, já tinham vivenciado situação de violência na infância. Além disso, em $60 \%$ dos casos, a violência acontece em ambos os espaços (público e doméstico) e os principais fatores que potencializam são os ciúmes, uso de álcool e drogas, traição e separação (Defensoria Pública Do Estado Do Ceará, 2019; Rolim de Holanda et al., 2018).

A literatura acerca dessa matéria aponta que, entre os fatores de risco para violência contra a mulher, estão: baixa escolaridade, uso de álcool e outras drogas, estado civil, cor, religião, saúde mental, número de filhos, falta de apoio familiar, abuso na infância, "sexarca" (sic) precoce, desemprego, dependência financeira, comportamento agressivo e/ou controlador por parte do parceiro, baixo nível econômico, aceitação ou subserviência, histórico de violência na família e situação ocupacional(Medeiros, 2015; Silva et al., 2015; Fiorotti et al., 2018).

Souza et al. (2019) expressam que o fenômeno da violência sofrida pelas mulheres está relacionado às diferenças culturais e comportamentais, bem como à visão do papel social da mulher, como inserção no mercado de trabalho, nível de escolaridade, número de filhos, religião, entre outros aspectos, que provocam influxos distintos no adoecimento e na morte, em decorrência da violência nessa população.

As relações conjugais, na sociedade machista, denotam intensivo potencial para promoção das violências no âmbito intrafamiliar. Relações violentas masculinas configuram-se como inerentes ao desemprego, resultando em desestruturação da identidade masculina e refletindo, de modo agressivo, em sua companheira, bem como se vinculam ao consumo de álcool e outras drogas. Embora exista o uso de drogas, como a maconha e a cocaína, por parte dos agressores, o álcool é o mais consumido no País, principalmente por homens, e que começam cada vez mais cedo a ingerir isso em decorrência do fato de ser aceito o consumo dessa droga pela sociedade, o que torna a substância mais nociva ao funcionamento familiar, uma vez que conduz a mudanças de humor, repercutindo em agressão física, não só para com suas parceiras, mas, também, para com os filhos (Medeiros, 2015).

No que concerne aos tipos de agressões, o estudo revelou que as mulheres sofrem predominantemente violência física, ao passo que, entre os mecanismos de ação mais utilizados, está o emprego da força corporal/espancamento, seguido do uso de objetos perfurocortantes. Esses dados foram apontados pelo Ministério da Saúde (2017) no último inquérito do Sistema de Vigilância de Violências e Acidentes (VIVA), em unidades de urgência e emergência pelo Brasil, no qual foi apontada a violência física $(98,5 \%)$ como a principal, sendo o expediente mais empregado a força corporal/espancamento (70,9\%), enquanto a natureza da lesão conforma o corte/laceração (35,8\%) (Garcia \& Silva, 2018).

O estudo demonstrou que, com o passar dos anos, houve mais notificação dos casos de violência contra a mulher, bem como aumento da taxa de mortalidade, sendo que a TM teve maior elevação em 2018 e redução nos anos de 2015 e 2016 . Aditase, por ser oportuno, o fato de que o Estado do Ceará dá um salto nas políticas públicas de enfretamento à violência contra a mulher em 2011, com o advento da Rede de Enfrentamento à Violência Contra a Mulher pela Secretaria de Políticas para Mulheres (SPM) da Presidência da República (Brasil \& Secretaria Nacional de Enfrentamento à Violência contra as Mulheres, 2011).

O sistema reúne ações e serviços de variados setores, em especial, da assistência social, da justiça, da segurança pública e da saúde, que visam i) à ampliação e melhoria da qualidade do atendimento; ii) à identificação e ao encaminhamento adequado das mulheres em situação de violência; e iii) à integridade e à humanização do atendimento às mulheres vítimas de violência (Brasil \& Secretaria Nacional de Enfrentamento à Violência contra as Mulheres, 2011).

O Estado do Ceará possui uma rede extensa para atendimento as vítimas em situação de violência, com eixos especializados no Judiciário, como a Casa da Mulher Brasileira, inaugurada em 14 de dezembro de 2018 (Araújo et al., 2018).

Outro equipamento relevante para o atendimento das mulheres configura as Unidades Móveis, em funcionamento desde 2015 no Ceará e, até setembro de 2018, 5.115 mulheres foram atendidas em grupos de conversas ou individualmente. Ressalta- 
se que a equipe é multidisciplinar e que o atendimento abrange desde prevenção, assistência, encaminhamento e acompanhamento, até práticas educativas, palestras e distribuição de material informativo sobre a Lei Maria da Penha (Araújo et al., 2018).

Essas ações já aconteceram em quase todos os municípios do Ceará, trazendo uma descentralização do acesso aos direitos e assistência às vítimas de violência (Araújo et al., 2018).

De acordo com Silva et al. (2018), além dessas ações, duas portarias estenderam o atendimento das delegacias especificas de defesa da mulher no Ceará para mulheres transexuais e travestis vítimas de violência (GDGPC n²31/2017 e GDGPC no 30/2017), independentemente do sujeito ativo do delito, com o objetivo de garantir a efetivação do direito a um atendimento humanizado e especializado, buscando minimizar as consequências da violência sofrida (Portaria GDGPC no 231, de 16 de outubro de 2017, 2017). Tais portarias consideram que o elemento diferenciador da Lei Maria da Penha é o gênero, haja vista que o sexo biológico e a identidade subjetiva nem sempre coincidem. De tal modo, sobra ratificada a defesa do atendimento diferenciado e qualificado dessas mulheres em situação de violência (Portaria GDGPC no 30, de 10 de março de 2017, 2017).

De acordo com a Defensoria Pública do Estado do Ceará, no período de 2016 a 2019, de 573 mulheres que sofreram violência e receberam assistência jurídica, psicológica e social da Defensoria, inseridas no contexto familiar de violência doméstica, 273 revelaram que não pretendem representar criminalmente contra o agressor (Defensoria Pública Do Estado Do Ceará, 2019).

Desde o ano de 2003, com a aprovação da Lei $n^{\circ} 10.778$, foi estabelecida a obrigatoriedade de notificação de casos de violência contra a mulher (Lei no 10.778, de 24 de novembro de 2003, 2003). Visando a atender essa obrigatoriedade, o Ministério da Saúde, em 2014, aprovou a Portaria GM/MS nº 1.271, que inclui a violência na Lista Nacional de Notificação Compulsória de doenças, agravos e eventos de saúde pública. A notificação, entretanto, ficava restrita ao sistema de saúde e possuía caráter sigiloso (Portaria no 1.271, de 6 de junho de 2014, 2014). No mês de dezembro de 2019, a lei 10.778 foi alterada pela Lei $\mathrm{n}^{\mathrm{o}}$ 13.931, ampliando a necessidade de notificação em 24 horas à autoridade policial, não apenas os casos confirmados, mas também as situações com indícios de violência (Lei no 13.931, de 10 de dezembro de 2019, 2019).

Nesse sentido, os profissionais de saúde, atuando em um serviço privado ou público de sua especialidade, possui a obrigação legal de comunicar, além da Vigilância em Saúde, também à polícia. O enfermeiro é um profissional que tem potencial para identificação precoce dos casos de violência sexual e atuar no tratamento dos pacientes por via de um atendimento mais humanizado e integral. Muitos enfermeiros, contudo, referem não estar preparados técnico-cientificamente para agir ante essas situações, e algumas pesquisas nacionais demonstraram essa realidade no curso de alguns anos (Cristiane Nunes de Souza et al., 2019).

\section{Conclusão}

A investigação que se termina de expor evidenciou algumas características da violência interpessoal sofrida pelas mulheres no Estado do Ceará. O perfil revela que são mulheres jovens, pardas, com baixa escolaridade, cujos companheiros são os agressores. Além disso, o local mais comum onde são acometidas pelo ato violento é o ambiente doméstico, principalmente pela violência física, por meio do uso de força e objetos perfurocortantes.

Os dados também exprimiram que, no decorrer dos anos, as taxas de incidência aumentaram, o que revela a efetividade de algumas políticas de saúde pública dirigidas a este rol de problemas, como o expediente da notificação compulsória nos serviços de saúde desde 2003. De outra parte, observou-se, também, o aumento das taxas de mortalidade no Estado, fato revelador do aumento constante dos casos de violência fatal contra esse grupo, também expressivo de que, embora desde 2006 tenham surgido mecanismos na legislação brasileira e na rede de proteção com o intuito de minimizar/resolver o problema, como a Lei 
Maria da Penha, estes não estão surtindo o efeito esperado. Faz-se necessário refletir, então, se as medidas adotadas não ocorrem, meramente, como de caráter repressor e pouco educativas, e até que ponto isso influencia no problema.

Este estudo apresenta limitações, sendo a principal o uso de dados secundários que está sujeito subnotificações, principalmente quanto ao preenchimento adequado dos dados e indexação destes no sistema de informação. Desta forma, sugerese a realização de estudos mais específicos com análises estatísticas mais robustas para melhor compreender o fenômeno abordado e sua interferência na sociedade.

\section{Referências}

Araújo, M. de L. G., Albuquerque, G. A., \& Medina, L. L. G. (2018). Prevenção e tratamento dos agravos resultantes da violência sexual contra mulheres e adolescentes (Vol. 2). Escola de Saúde Pública do Ceará. https:/www.esp.ce.gov.br/wp-content/uploads/sites/78/2019/05/Caderno-Viol\%C3\%AAncia-Contraa-Mulher-Digital.pdf

Brasil \& Departamento de Informática do Sistema Único de Saúde. (2020). Morbidade Hospitalar do SUS. http://www2.datasus.gov.br/DATASUS/index.php?area=0205

Brasil \& Fórum Brasileiro de Segurança Pública. (2019). Atlas da violência 2019. http://www.forumseguranca.org.br

Brasil \& Ministério da Saúde. (2012). Prevenção e tratamento dos agravos resultantes da violência sexual contra mulheres e adolescentes (3 ${ }^{\circ}$ ed). Ministério da Saúde. https://bvsms.saude.gov.br/bvs/publicacoes/prevencao_agravo_violencia_sexual_mulheres_3ed.pdf

Brasil \& Secretaria Nacional de Enfrentamento à Violência contra as Mulheres. (2011). Politica Nacional de Enfrentamento à Violência Contra as Mulheres. Ministério da Justiça.

Defensoria Pública Do Estado Do Ceará. (2019). Defensoria Pública divulga perfil de mulheres vitima de violência doméstica que buscaram assistência. http://www.defensoria.ce.def.br/noticia/defensoria-publica-divulga-perfil-de-mulheres-vitima-de-violencia-domestica-que-buscaram-assistencia

Fiorotti, K. F., Amorim, M. H. C., Lima, E. de F. A., Primo, C. C., Moura, M. A. V., \& Leite, F. M. C. (2018). Prevalência E Fatores Associados À Violência Doméstica: Estudo Em Uma Maternidade De Alto Risco. Texto \& Contexto - Enfermagem, 27. https://doi.org/10.1590/0104-07072018000810017

Garcia, L. P., \& Silva, G. D. M. da. (2018). Violência por parceiro íntimo: Perfil dos atendimentos em serviços de urgência e emergência nas capitais dos estados brasileiros, 2014. Cadernos de Saúde Pública, 34, e00062317. https://doi.org/10.1590/0102-311x00062317

Instituto De Pesquisa Econômica Aplicada. (2020). Atlas da violencia. https://www.ipea.gov.br/atlasviolencia/publicacoes

Lei $n^{\circ} 10.778$, de 24 de novembro de 2003. Estabelece a notificação compulsória, no território nacional, do caso de violência contra a mulher que for atendida em serviços de saúde públicos ou privados. (2003) (testimony of Brasil), Diário Oficial da União. http://www.planalto.gov.br/ccivil_03/LEIS/2003/L10.778.htm

Lei $n^{\circ} 13.931$, de 10 de dezembro de 2019. Altera a Lei n ${ }^{\circ} 10.778$, de 24 de novembro de 2003, para dispor sobre a notificação compulsória dos casos de suspeita de violência contra a mulher (2019) (testimony of Brasil), Diário Oficial da União.

Lima-Costa, M. F., \& Barreto, S. M. (2003). Tipos de estudos epidemiológicos: Conceitos básicos e aplicações na área do envelhecimento. Epidemiologia e Serviços de Saúde, 12(4). https://doi.org/10.5123/S1679-49742003000400003

Medeiros, M. N. (2015). Avaliação de risco em casos de violência contra a mulher perpetrada por parceiro íntimo. https://doi.org/10.26512/2015.02.T.20191

Paraná \& Secretaria de Estado da Saúde do Paraná. (2017). Protocolo para o atendimento às pessoas em situação de violência sexual (2a ed). SESA. https://bvsms.saude.gov.br/bvs/publicacoes/prevencao_agravo_violencia_sexual_mulheres_3ed.pdf

Portaria GDGPC $n^{\circ}$ 231, de 16 de outubro de 2017. Amplia o Atendimento Especializado nas Delegacias de Defesa da Mulher (DDM'S), no Estado do Ceará, às Mulheres vítimas de violência sexual, independentemente do sujeito ativo do delito. (2017) (testimony of Ceará), Diário Oficial da União. https://www.jusbrasil.com.br/diarios/168792441/doece-20-11-2017-pg-45

Portaria GDGPC n 30, de 10 de março de 2017. Amplia o Atendimento Especializado nas Delegacias de Defesa da Mulher (DDM'S), no Estado do Ceará, às Mulheres Travestis e Transexuais em situação de violência doméstica e familiar, prevista na Lei 11.340/2006. (2017) (testimony of Ceará), Diário Oficial da União. https://www.policiacivil.ce.gov.br/wp-content/uploads/sites/26/2018/01/port-30.pdf

Portaria $n^{o}$ 1.271, de 6 de junho de 2014. Define a Lista Nacional de Notificação Compulsória de doenças, agravos e eventos de saúde pública nos serviços de saúde públicos e privados em todo o território nacional, nos termos do anexo, e dá outras providências. (2014) (testimony of Brasil), Diário Oficial da União.

Rolim de Holanda, E., Rolim de Holanda, V., Silva de Vasconcelos, M., Patriota de Souza, V., \& Teresinha Gimeniz Galvão, M. (2018). Fatores associados à violência contra as mulheres na atenção primária de saúde. Revista Brasileira em Promoção da Saúde, 31(1), 1-9. https://doi.org/10.5020/18061230.2018.6580

Silva, C. D., Gomes, V. L. de O., Mota, M. S., Gomes, G. C., \& Amarijo, C. L. (2015). Violence against women: Aggressors drug users. Revista de Pesquisa: Cuidado é Fundamental Online, 7(2), 2494. https://doi.org/10.9789/2175-5361.2015.v7i2.2494-2504

Souza, C. N. de, Silva, J. de S., Carvalho, N. R. B. de, Aoyama, E. de A., \& Lima, R. N. (2019). O Papel Da Enfermagem Na Violência Sexual Contra A Mulher. ReBIS, 1(4), 31-36.

Souza, M. C. (2009). A Convenção sobre a Eliminação de todas as Formas de Discriminação contra as Mulheres e suas implicações para o direito brasileiro. Revista eletrônica de Direito Internacional, 5(1), 346-386 